\title{
DESENVOLVIMENTO E TESTES DE MINI-SONDA WENNER PARA AVALIAÇÃO DE CONTAMINAÇÕES RASAS
}

\author{
Fabio Taioli ${ }^{1}$, José Domingos Faraco Gallas², Vilmondes Ribeiro³, \\ Patrícia Braga Toledo lezzi ${ }^{4}$ e Diego Potomati Vaccaro Nascimento ${ }^{5}$ \\ Recebido em 2 agosto, 2006 / Aceito em 27 dezembro, 2006 \\ Received on August 2, 2006 / Accepted on December 27, 2006
}

\begin{abstract}
The evaluation of soil and groundwater contamination has been an important task in the geosciences field and pushing on the methodological improvement. The contamination extension is normally investigated using geophysical methods or boreholes and chemical analysis. Otherwise, several contamination problems are at low depths (less than $0.5 \mathrm{~m}$ ) allowing the easy access to the contaminated region. Therefore, a low-cost and simple system (mini-probe Wenner) was developed and tested and it is aimed at low depth resistivity measurements. The system is formed by a current source (AC) and a PVC probe which contains a Wenner array with a electrode spacing of $1 \mathrm{~cm}$. Laboratory tests show that the current source works adequately in the resistance range normally associated with contaminated materials while the system tests (including the current source and probe) indicated apparent resistivity values very similar to the resistivity values calculated using the equation proposed by Archie, in the 1.5 to $57 \Omega \mathrm{m}$ interval. Preliminary field tests showed that the mini-probe is easily applicable in sandy and sand-silty soils while in clayey or hard soils is difficult its introduction. Therefore, interesting applications to the proposed system are the study of agricultural areas or the study of contamination in predominantly sandy lithologies.
\end{abstract}

Keywords: electroresistivity, contamination, mini-probe, salinization.

RESUMO. 0 estudo e avaliação de contaminações do solo e/ou água subterrânea tem sido uma das grandes preocupações na área de geociências, exigindo constante desenvolvimento metodológico. A definição da extensão da contaminação é normalmente efetuada por métodos indiretos, particularmente os geofísicos (eletroresistividade, eletromagnético e GPR), e por métodos diretos (sondagens, poços de monitoramento, análises químicas etc.). No entanto, muitos dos problemas de contaminação são bastante rasos (menos de 0,5 metro de profundidade) permitindo o fácil acesso à região contaminada. Neste sentido foi desenvolvido e testado um sistema simples (mini-sonda Wenner) e de baixo custo para medidas de resistividade in-situ a pequenas profundidades. 0 sistema é composto por uma fonte de corrente alternada e uma ponteira de PVC sobre a qual é instalado um arranjo Wenner com espaçamento entre eletrodos de $1 \mathrm{~cm}$. Testes de bancada mostraram que a fonte de corrente se comporta satisfatoriamente na faixa de resistências normalmente associadas a materiais contaminados, enquanto que os testes laboratoriais do sistema (fonte e sonda) levaram a valores de resistividade aparente medidos muito próximos dos valores de resistividade calculados utilizando-se a equação proposta pela Lei de Archie, no intervalo de 1,5 $\Omega$.m a $57 \Omega$.m. Testes preliminares de campo mostraram que a mini-sonda é facilmente utilizável em solos arenosos e areno-siltosos, porém, em solos mais argilosos ou demasiadamente compactados torna-se difícil a sua introdução. Desta forma, aplicações interessantes para o sistema proposto podem ser 0 estudo de salinização de áreas agrícolas, onde normalmente se tem solo arado e fofo, ou estudo de contaminações rasas em litologias predominantemente arenosas.

Palavras-chave: eletroresistividade, contaminação, mini-sonda, salinização.

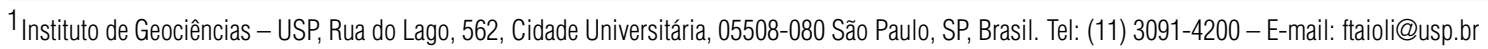

2 Instituto de Geociências - USP, Rua do Lago, 562, Cidade Universitária, 05508-080 São Paulo, SP, Brasil. Tel: (11) 3091-4232 - E-mail: jgallas@usp.br

3 Instituto de Pesquisas Tecnológicas - IPT, Av. Prof. Almeida Prado, 532, Cidade Universitária, 05508-080 São Paulo, SP, Brasil. Tel: (11) 3767-4932

-E-mail: vilmonds@ipt.br

${ }^{4}$ Instituto de Geociências - USP, Rua do Lago, 562, Cidade Universitária, 05508-080 São Paulo, SP, Brasil - E-mail: patriciaiezzi@yahoo.com.br

5 Instituto de Geociências - USP, Rua do Lago, 562, Cidade Universitária, 05508-080 São Paulo, SP, Brasil - E-mail: diegopvn@gmail.com
} 


\section{INTRODUÇÃO}

Devido principalmente à sua importância econômica e social, 0 estudo e avaliação de contaminações do solo e/ou água subterrânea tem sido uma das grandes preocupações na área de geociências e tem exigido constante desenvolvimento metodológico. A extensão tanto em área como em profundidade de contaminações por produtos orgânicos ou inorgânicos é normalmente definida primeiramente por métodos indiretos, particularmente os geofísicos (eletroresistividade, eletromagnético e GPR), e por métodos diretos (sondagens, poços de monitoramento, análises químicas etc.). 0 uso de técnicas indiretas depende de uma série de fatores, tais como a profundidade de interesse, resolução desejada, constituição mineralógica e grau de saturação do solo, interferências presentes na área de estudo, entre outros. Desta forma, muitas vezes sua aplicação torna-se prejudicada ou mesmo inviável. Por outro lado, muitos dos problemas de contaminação encontram-se a pequenas profundidades (menos de 0,5 metro de profundidade) permitindo o fácil acesso à região contaminada. Porém, a implantação de uma rede de poços de monitoramento pode atingir um custo proibitivo para muitos projetos devido à necessidade de manutenção dos poços, constante amostragem e análises químicas. Este trabalho apresenta 0 desenvolvimento e testes de uma pequena sonda que possibilita a medida da resistividade do solo seco ou saturado in situ, a pequenas profundidades, sem que seja necessária a instalação de poços de monitoramento, portanto, permitindo medidas rápidas e a extremo baixo custo. Consiste em uma sonda dotada de quatro eletrodos dispostos segundo o arranjo Wenner em conjunto com uma fonte de corrente alternada e dois multímetros, o que torna possível a execução de uma medida convencional de resistividade em diferentes profundidades à medida que a sonda é cravada no solo. Desta forma é possível executar-se medidas em diferentes profundidades e se obter a seção de distribuição de resistividade próxima à superfície (até cerca de 1,5 m de profundidade, dependendo do tipo de solo). A vantagem desta técnica é o baixíssimo custo, tanto do sistema de medidas como do custo operacional e simplicidade na análise e interpretação dos resultados.

\section{REVISÃO BIBLIOGRÁFICA}

A concepção da mini-sonda Wenner para as medidas in situ baseia-se nas medidas de resistividade no eletrólito que constitui o subsolo e seu conteúdo iônico. A condução da corrente elétrica através de íons (condução iônica ou eletrolítica) é, sem dúvida, o processo de condução de maior importância e interesse na prospecção por eletroresistividade (Orellana, 1972).
Uma vez que a grande maioria das rochas e solos são maus condutores, suas resistividades deveriam ser extremamente altas, não fosse pelo fato que usualmente elas apresentam interstícios que estão preenchidos por fluidos, principalmente água (Gallas, 2000).

Em vista disso, efetivamente as rochas (e o subsolo em geral) assumem um comportamento de condutores eletrolíticos (Telford et al., 1990) e a propagação da corrente processa-se por condução iônica, através de moléculas que tenham excesso ou deficiência de elétrons.

As resistividades também variam com a mobilidade, concentração e grau de dissolução dos íons, sendo que o grau de dissociação depende da constante dielétrica da solução. Comparativamente à condução ôhmica (eletrônica), a condução iônica não é tão somente mais lenta, mas representa também um transporte de material (Keller \& Frischknecht, 1977).

Assim, na grande maioria das litologias encontradas na natureza, a condução será eletrolítica, sendo o meio condutor uma solução de água e sais comuns distribuídos de maneira complexa na estrutura dos poros da rocha. A resistividade dependerá da quantidade de água presente, da salinidade da mesma e a maneira como a água distribui-se na rocha.

A expressão salinidade equivalenteé quase sempre utilizada quando se trata de resistividade da água. Como normalmente a água contém uma grande variedade de sais em solução, é bastante difícil obter-se a resistividade de uma amostra de água, a partir de uma análise química. Objetivando facilitar a tarefa, a salinidade equivalente de uma solução é definida como a salinidade de uma solução de $\mathrm{NaCl}$ que teria a mesma resistividade de uma solução para a qual a salinidade equivalente está sendo expressa (Keller \& Frischknecht, 1977). A salinidade equivalente pode ser considerada como verdadeira, uma vez que as mobilidades dos íons presentes na grande maioria das soluções não variam significativamente.

A utilização do conceito de salinidade equivalente permite a simplificação do processo de cálculo da resistividade de uma solução. A Figura 1 ilustra o comportamento da resistividade em função da concentração de um ácido e de dois tipos de sais, particularmente do $\mathrm{NaCl}$ nas temperaturas de $18^{\circ} \mathrm{C}$ e $100^{\circ} \mathrm{C}$.

Como pode ser observado na Figura 1, o comportamento da resistividade em função da concentração de ambos sais é praticamente linear, até a concentração de 100g/L. A Figura 1 também permite observar, através das duas curvas referentes ao $\mathrm{NaCl}$ nas temperaturas de $18^{\circ} \mathrm{C} \mathrm{e} 100^{\circ} \mathrm{C}, 0$ comportamento decrescente da resistividade com 0 aumento da temperatura. 


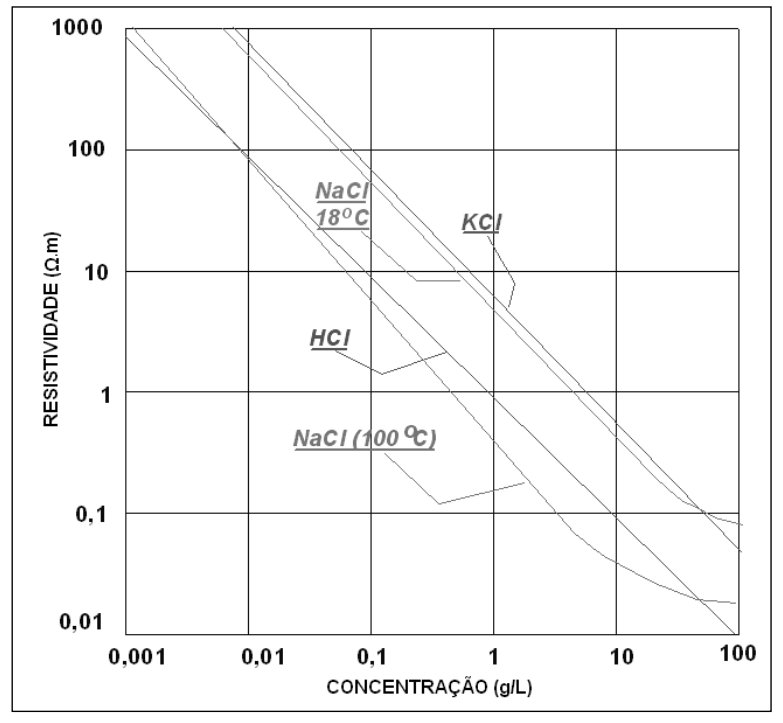

Figura 1 - Relação entre resistividade e concentração de substâncias em solução à temperatura de $18^{\circ} \mathrm{C}$ e $\mathrm{NaCl}$ também a $100^{\circ} \mathrm{C}$ (adaptado de Keller \& Frischknecht, 1977).

\section{POROSIDADE E TEXTURA DA ROCHA COM RELAÇÃO À RESISTIVIDADE}

A resistividade de uma rocha decresce com 0 aumento de seu conteúdo de água e também com o volume dissolvido de cloretos, sulfatos (sais em geral) e presença de outros minerais. Em rochas totalmente saturadas, o conteúdo de água pode ser calculado pela sua porosidade. Em rochas parcialmente saturadas, 0 efeito de dessaturação sobre a resistividade precisa ser considerado. A textura da rocha também tem efeito sobre a resistividade.

Para haver boa condução elétrica, os poros precisam estar interligados e preenchidos com água. 0 volume total dos poros é constituído de duas partes: poros de armazenamento e poros de conexão. Uma rocha com elevada razão entre poros de armazenamento e poros de conexão apresentará uma resistividade maior do que uma rocha com idêntica porosidade, mas com menor taxa de armazenamento/conexão entre poros.

No caso de argilas, os poros não estão interligados (baixa permeabilidade), mas as mesmas costumam apresentar baixas resistividades, desde que contenham água, porém isto se deve a fenômenos de troca iônica (Gallas, 2000).

Muitos trabalhos têm sido feitos no sentido de correlacionarse resistividade com conteúdo de água, inferindo-os para rochas que contém petróleo (Keller \& Frischknecht, 1977; Telford et al., 1990). Para estas rochas, que eram primariamente arenitos e calcários porosos, foi observado que a resistividade varia aproximadamente com 0 inverso do quadrado da porosidade, quando a rocha está totalmente saturada com água (Archie, 1942, apud
Telford et al., 1990). Esta observação levou ao uso difundido de uma relação empírica de resistividade e porosidade, conhecida como Lei de Archie.

$$
\rho=a \rho_{w} \varphi^{-m}
$$

onde:

$\rho=$ resistividade "bulk" (volumétrica);

$\rho_{w}=$ resistividade da água dos poros;

$\varphi=$ porosidade expressa como uma fração por unidade de volume;

$a \rightarrow$ relativo à porosidade da rocha, sendo ligeiramente menor que 1 para rochas com porosidade intergranular e ligeiramente maior que 1 para rochas com "porosidade" de juntas;

$m \rightarrow$ é o parâmetro de cimentação, sendo um pouco maior que 2 para rochas cimentadas e bem classificadas e um pouco menor que 2 para rochas mal classificadas e mal cimentadas.

Os valores para $a$ e $m$ referidos acima são os adotados por Keller \& Frischknecht (1977).

A Lei de Archie supõe que os poros das rochas estejam completamente saturados por água e que o volume de água contido reflita a porosidade. Isto nem sempre é verdadeiro, uma vez que os poros podem conter ar, gás ou mesmo petróleo. Desse modo, torna-se necessário introduzir o índice de resistividade, que é a relação entre a resistividade da rocha com o grau de saturação considerado e com os poros efetivamente preenchidos com água. Define-se como grau de saturação $S$ a fração de volume ocupada por água em relação ao volume total dos poros. Assim a resistividade da rocha será dada por:

$$
\rho=a b \varphi^{-m} S^{-n} \rho_{w}
$$

sendo:

$n \cong 2$ (Telford et al., 1990) e

$b=0,6$ e $n=2,25$ para arenitos e calcários arenosos de porosidade $40 \%$;

$b=0,4$ e $n=2,1$ para rochas carbonáticas de porosidade 25\% (Orellana, 1972).

\section{SENSOR}

A sonda para medidas de resistividade foi baseada em conceito apresentado por Stieglitz (2003) e desenvolvida empregando um pequeno arranjo Wenner (comumente utilizado em levantamentos geoelétricos de superfície). A sonda, construída sobre uma base de PVC, consiste em quatro anéis de fio de cobre espaçados entre si por $0,01 \mathrm{~m}$, sendo 0 diâmetro do fio de $1 \mathrm{~mm}$ (Figura 2). É rosqueada na ponta de um tubo de aço galvanizado, que é gra- 
dualmente inserido no solo por percussão, até cerca de 1,5m, se 0 solo permitir.

Através de cabos elétricos instalados internamente, tanto no tubo como na sonda, a corrente é fornecida aos anéis externos (AB) e a medida da diferença de potencial é efetuada entre os anéis centrais (MN). Para o fornecimento de corrente foi desenvolvido um pequeno circuito eletrônico (Figura 3) e as leituras de corrente e tensão são efetuadas em dois multímetros digitais comumente encontrados no mercado (Figura 4). 0 valor de resistividade aparente pode ser calculado pela equação derivada da Lei de Ohm, lembrando-se que em situações onde o conjunto eletródico encontra-se inserido no meio de medida deve-se utilizar a seguinte equação:

$$
\begin{aligned}
& \rho_{a}=K \frac{\Delta V}{I} \quad \text { sendo } \\
& K=\frac{4 \pi}{\frac{1}{A M}-\frac{1}{A N}-\frac{1}{B M}+\frac{1}{B N}}
\end{aligned}
$$

onde:

$\rho_{a}=$ resistividade aparente $(\Omega . m)$;

$\Delta V=$ diferença de potencial medida entre os eletrodos $M$ e $N(V)$;

$I=$ corrente aplicada no circuito (A);

$K=$ constante geométrica dependente do arranjo eletródico utilizado;

$A M, A N, B M$ e $B N=$ distâncias entre os respectivos eletrodos $(m)$.

Para a mini-sonda, que utiliza um arranjo tipo Wenner com distâncias entre eletrodos de 0,01m, o valor utilizado para a constante geométrica K foi de 0,12566.

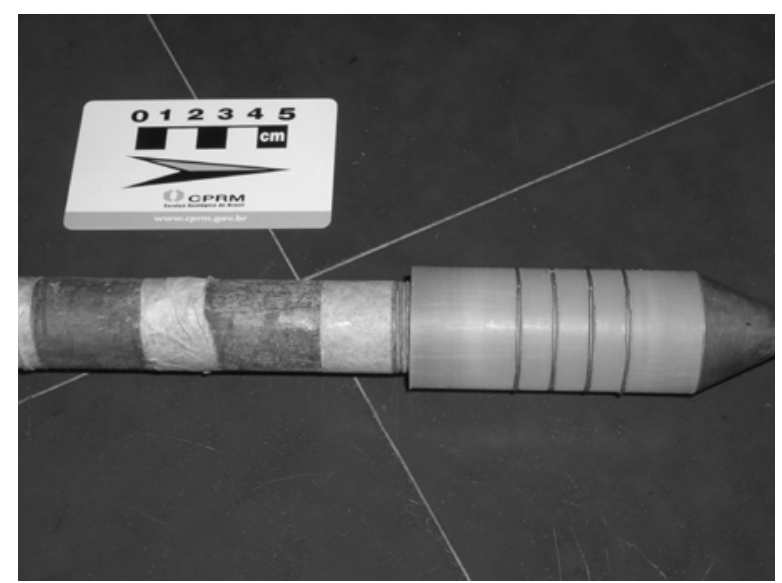

Figura 2 - Sensor de resistividade com mini-arranjo Wenner.

\section{CIRCUITO MINI-SONDA}

0 diagrama esquemático do sistema é apresentado na Figura 3 e é composto de uma fonte de alimentação (circuito 1) e de um oscilador de onda senoidal (circuito 2).

0 objetivo do circuito 1 (fonte de alimentação) é 0 de gerar as tensões positivas $(+5 \mathrm{~V})$ e negativas $(-5 \mathrm{~V})$, para alimentar 0 circuito oscilador de onda senoidal. Constitui-se basicamente de um regulador de tensão positiva, que gera os $+5 \mathrm{~V}$ a partir da tensão de entrada que pode variar de $8 \mathrm{~V}$ a $35 \mathrm{~V}$ e um regulador de tensão negativa, que gera os $-5 \mathrm{~V}$ a partir da tensão de entrada que também pode variar de $-8 \mathrm{~V}$ a $-35 \mathrm{~V}$.

0 objetivo do circuito 2 (oscilador de onda senoidal) é 0 de gerar o sinal senoidal, que será injetado nos eletrodos $A$ e $B$ da sonda Wenner. Trata-se um oscilador de onda senoidal, denominado de duplo-T, cuja freqüência de oscilação é dada por:

$$
f=\frac{1}{2 \pi R C}
$$

onde: $f$ é a frequiência de oscilação $(\mathrm{Hz}), R$ é 0 valor do resistor $(\Omega)$ e $C$ o valor do capacitor (F).

Este circuito é composto por um único elemento ativo, um amplificador operacional OPA 548, que foi escolhido por permitir uma grande variação da impedância de carga, além de permitir um ajuste simples da corrente máxima de saída.

Esta corrente pode ser ajustada, de zero a $5 \mathrm{~A}$, através de um resistor ou potenciômetro conectado entre os pinos 3 e 4 do OPA 548. Difere de outros amplificadores operacionais que normalmente utilizam um resistor em série com a corrente de saída para efetuar este controle. Esta solução foi adotada visando minimizar 0 consumo de baterias.

Os resistores $2 \mathrm{k} \Omega$ e $500 \Omega$ devem ser ajustados de modo que 0 circuito entre em oscilação.

0 sistema desenvolvido para este trabalho teve seus resistores e capacitores calculados para uma freqüência nominal de $1200 \mathrm{~Hz}$, freqüência esta que se encontra na faixa operacional da maioria dos multímetros convencionais.

Para alimentação do sistema foram utilizadas duas baterias de $9 \mathrm{~V}$, formando uma fonte simétrica.

\section{TESTES DE BANCADA}

Efetuaram-se testes de bancada colocando-se resistores padrões na saída da fonte de corrente e as medidas foram feitas conforme mostrado na Figura 5, utilizando-se um multímetro para medida de corrente e outro multímetro para medida da diferença de potencial através do resistor padrão. Calculou-se o valor de resistência medido utilizando-se a Lei de Ohm. Os resultados obtidos são 
(1)



(2)

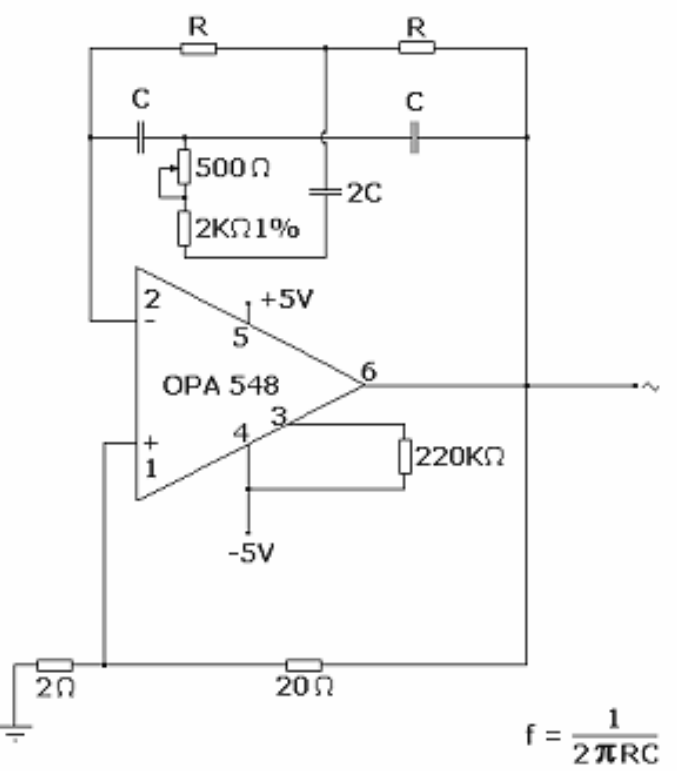

$\mathrm{f} @ 1200 \mathrm{~Hz}$

Figura 3 - Diagrama esquemático do circuito de fornecimento de corrente elétrica para o sensor. (1) fonte de alimentação; (2) oscilador de onda senoidal.

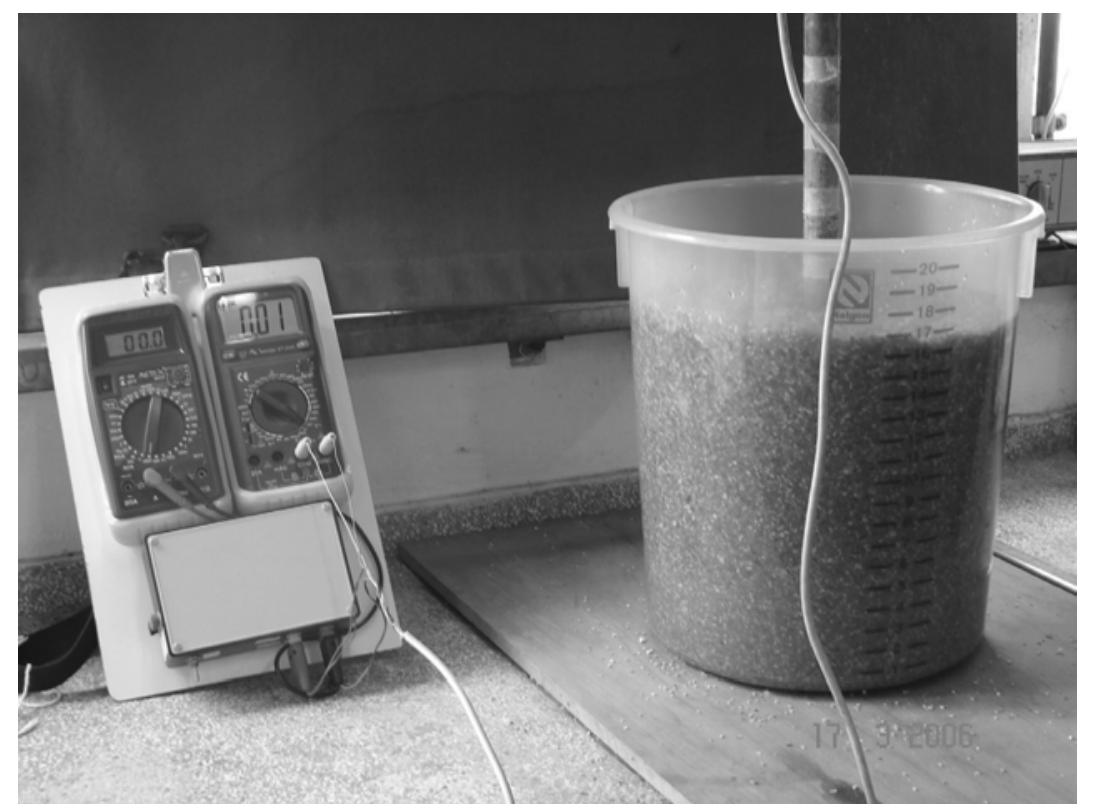

Figura 4 - Sistema completo, com a fonte de corrente, multímetros de leitura e sonda.

apresentados na Figura 6. Observou-se que para resistências com valores de até $100 \Omega$ os erros foram menores do que $6 \%$, enquanto para resistores até $5.000 \Omega$ a diferença entre 0 valor medido e o real não ultrapassou 10\%; a partir de $5.000 \Omega$ o valor medido tendeu a ser bem maior do que o real, atingindo um erro de cerca de 30\% para resistências de $100.000 \Omega$. Tendo em vista que a grande maioria dos materiais geológicos apresenta resistividades inferiores a $5000 \Omega$ e que a perspectiva de uso da sonda é para trabalhos em áreas contaminadas onde as resistividades raramente ultrapassam $100 \Omega$.m, considerou-se aceitável o com- 


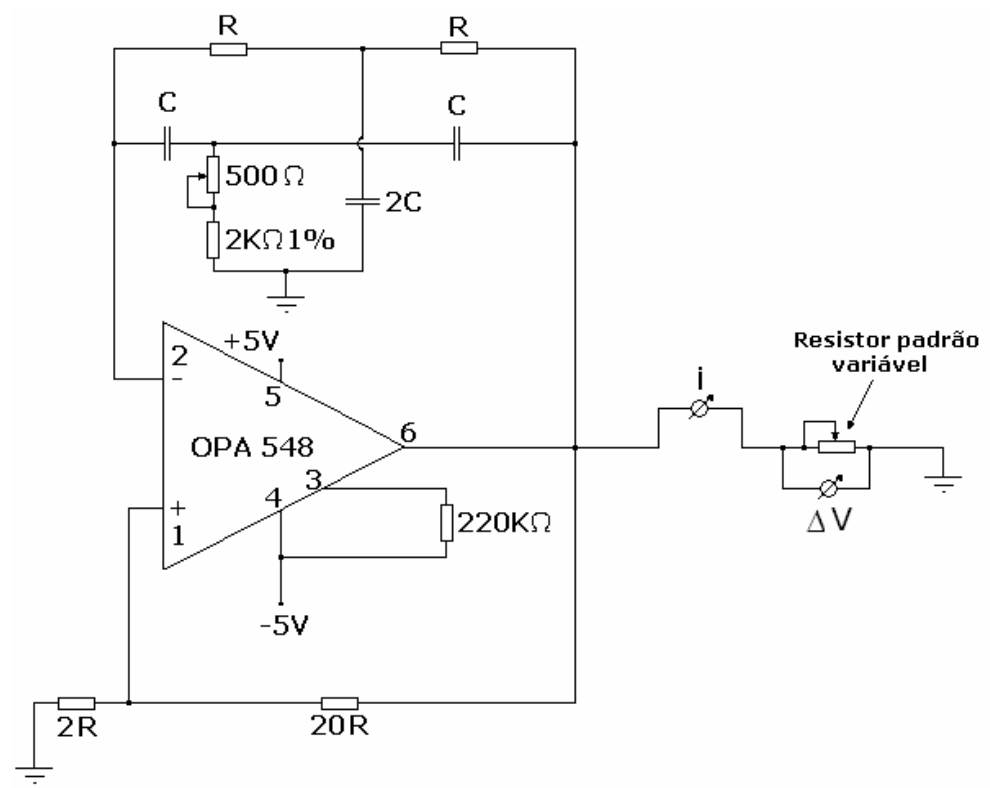

Figura 5 - Diagrama esquemático do circuito utilizado para os testes de bancada da fonte de corrente, com resistores padrões.

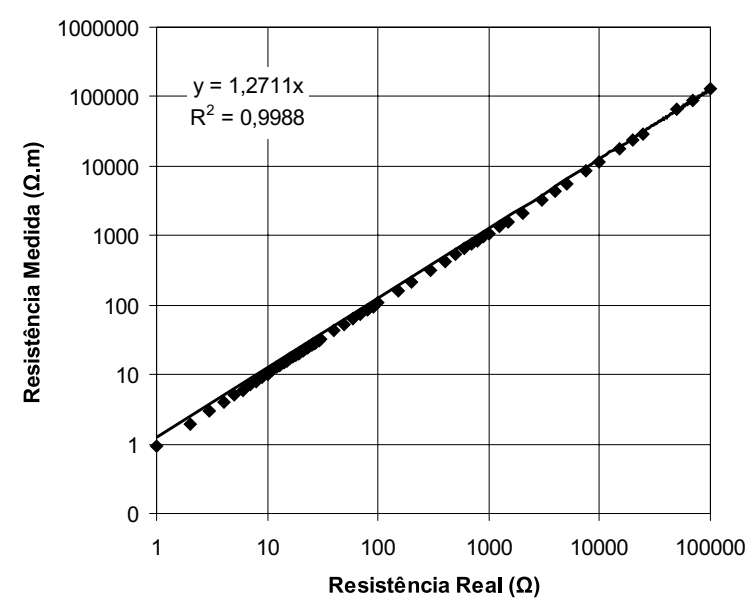

Figura 6 - Resultados de teste de bancada do circuito desenvolvido, comparando-se resistências padrões com os valores medidos e calculados.

portamento da fonte de corrente. No entanto, caso medidas mais precisas sejam necessárias, poderá ser efetuada a correção baseada na curva e respectiva equação obtidas no teste de bancada.

\section{PREPARAÇÃO DAS AMOSTRAS}

Para os ensaios em amostras simulando as condições de solo utilizou-se areia padrão (areia média a areia grossa), normalmente usada em pré-filtros de poços tubulares, que foi quarteada até se atingir porções com volume aproximado de 16 litros. A porosidade da amostra foi determinada por meio da medida da densi- dade do material $\left(\rho_{m}\right.$ - massa do material/volume de água deslocado em uma proveta), determinação da densidade da amostra ( $\rho_{a}$ - massa/volume) e finalmente cálculo da porosidade $(\varphi)$ utilizando a equação:

$$
\varphi=\frac{\rho_{m}-\rho_{a}}{\rho_{m}-\rho_{a}}
$$

onde $\rho_{a r}$ é a densidade do ar.

0 valor de porosidade obtido para a referida amostra foi de 0,409 .

Para preparação do eletrólito foram escolhidas dez dife- 
rentes concentrações de solução de $\mathrm{NaCl}$, para a realização das medidas efetuadas com a sonda de eletroresistividade. Como concentração inicial escolheu-se o que seria em média a concentração da água do mar, ou seja, $30 \mathrm{~g} / \mathrm{L}$ de $\mathrm{NaCl}$, e foi fracionada em dez partes. Porém, após o primeiro experimento com a décima parte dessa concentração ( $3 \mathrm{~g} / \mathrm{L} \mathrm{de} \mathrm{NaCl}$ ) observou-se que concentrações acima desse valor exigiriam demasiada corrente da fonte de corrente. Decidiu-se, então, fracionar em doze partes a concentração do primeiro experimento.

As concentrações de $\mathrm{NaCl}$ utilizadas foram as seguintes: 3,0g/L; 2,7g/L; 2,4g/L; 2,1g/L; 1,8g/L; 1,5g/L; 1,2g/L; 0,9g/L; $0,6 \mathrm{~g} / \mathrm{L} ; 0,3 \mathrm{~g} / \mathrm{L}, 0,15 \mathrm{~g} / \mathrm{L}$ e $0,075 \mathrm{~g} / \mathrm{L}$.

A condutividade elétrica da solução foi medida utilizando-se um condutivímetro modelo Multiline P3 pH/LF-SET. A temperatura ambiente do laboratório variou entre $21^{\circ} \mathrm{C}$ e $25^{\circ} \mathrm{C}$. Os vaIores de condutividade elétrica assim obtidos foram convertidos para resistividade elétrica e utilizados na equação (1) $\operatorname{como} \rho_{w}$, ou seja, resistividade da água dos poros.

\section{RESULTADOS OBTIDOS}

A Figura 7 apresenta os resultados da condutividade elétrica da solução em função da concentração de $\mathrm{NaCl}$ utilizada.

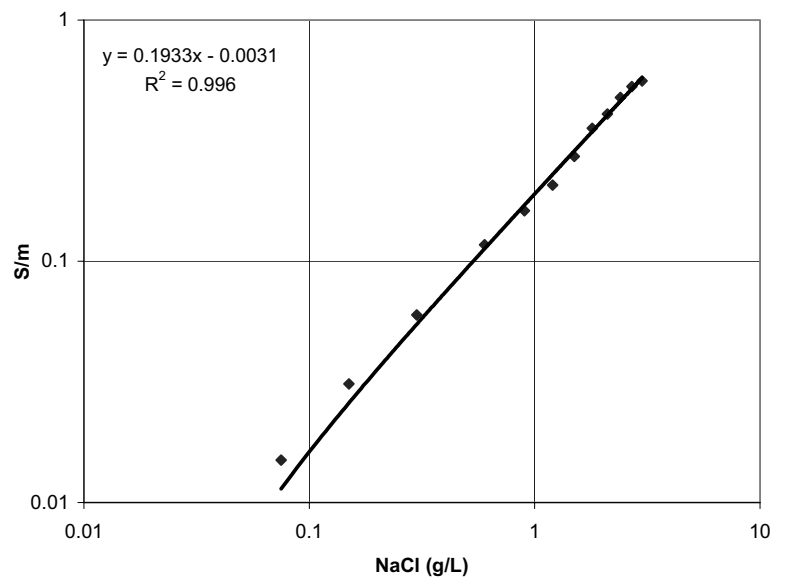

Figura 7 - Condutividade elétrica da solução em função da concentração de $\mathrm{NaCl}$ (medidas efetuadas em laboratório).

As soluções foram colocadas em recipiente com areia até a saturação da amostra. Inseriu-se, então, a mini-sonda na amostra até as profundidades de 5, 10 e $15 \mathrm{~cm}$, efetuando-se leituras dos valores de corrente elétrica e diferença de potencial e calculandose as resistividades aparentes para cada uma das profundidades.

Os valores obtidos foram utilizados na equação 1 (Lei de Archie) de forma a se obter a resistividade teórica. Utilizaram-se também os valores de $\mathrm{a}=0,351$ e $\mathrm{m}=1$, sendo que 0 valor de a foi calculado de forma a se obter o melhor ajuste para 0 conjunto dos dados, enquanto o valor de $\mathrm{m}$ foi atribuído considerandose a amostra utilizada (mal classificada e mal cimentada). Os resultados obtidos são apresentados na Figura 8, e evidenciam uma ótima concordância entre os valores obtidos e os calculados utilizando-se a Lei de Archie (coeficiente de Pearson = 0,996). Os desvios que ocorreram podem ser atribuídos, em parte, a sais impregnados na própria areia utilizada para os experimentos, uma vez que as amostras não foram lavadas para a execução dos ensaios.

A Figura 9 apresenta os dados de resistividade aparente obtidos com a mini-sonda em função da concentração da solução de $\mathrm{NaCl}$. Observa-se que as medidas permitiram um ótimo ajuste, com coeficiente de Pearson $=0,995$. A Figura 10 apresenta os valores obtidos inseridos na Figura 1 (Keller \& Frischknecht, 1977), onde pode-se observar que os resultados acompanharam a tendência dos gráficos propostos por aqueles autores.

\section{CONCLUSÕES}

Foi desenvolvido e testado um sistema simples e de baixo custo para medidas de resistividade in-situ a pequenas profundidades. 0 objetivo desta mini-sonda é sua aplicação em áreas contaminadas rasas, possíveis de serem atingidas introduzindo-se a sonda por percussão manual. 0 sistema é composto por uma fonte de corrente alternada e uma ponteira de PVC sobre a qual é instalado um arranjo Wenner com espaçamento entre eletrodos de $1 \mathrm{~cm}$. Esta ponteira é presa a um tubo galvanizado com diâmetro de 3/4" (19,05 mm), sendo que os cabos condutores passam pelo seu interior. Os testes de laboratório sobre o circuito da fonte de corrente evidenciaram erros menores do que $10 \%$ para valores de resistências até $5000 \Omega$, enquanto para resistências até $100 \Omega$ os erros foram inferiores a $6 \%$. Para resistências de valores mais elevados o erro aumenta (atingindo até 30\%). No entanto, considerou-se os erros aceitáveis, visto que solos contaminados normalmente apresentam resistividades aparentes inferiores a $100 \Omega$.m.

Em testes utilizando areia e soluções padrões de $\mathrm{NaCl}$ os valores de resistividade aparente medidos foram muito próximos dos valores de resistividade calculados utilizando-se a equação proposta pela Lei de Archie, no intervalo de $1,5 \Omega$.m a $57 \Omega$.m. Apesar de não terem sido efetuadas medidas em amostras saturadas com soluções de menores concentrações, esta faixa de valores, na maioria das situações, é compatível com os valores de resistividade observados em áreas contaminadas. Os valores de resistividade obtidos foram consistentes com as concentrações das soluções utilizadas quando comparadas com os valores 


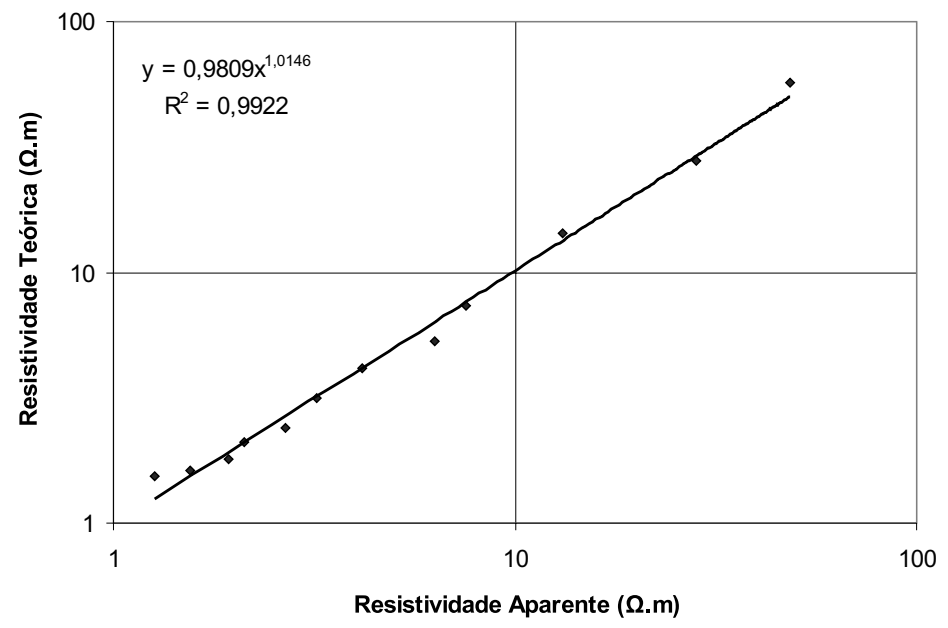

Figura 8 - Comparação entre os valores medidos de resistividade utilizando a mini-sonda e os calculados segundo a Lei de Archie.

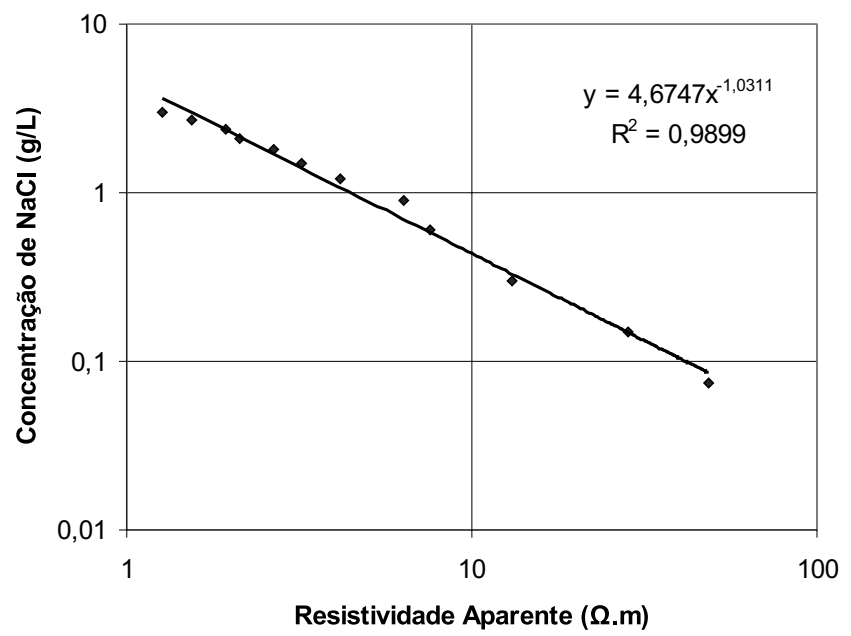

Figura 9 - Resistividade aparente obtida com a mini-sonda em função da concentração de NaCl da solução.

observados por Keller \& Frischknecht (1977).

Apesar de não ser escopo do presente trabalho, medidas preliminares de campo mostraram que a mini-sonda é facilmente utilizável em solos arenosos e areno-siltosos. Porém, em soIos mais argilosos ou demasiadamente compactados torna-se difícil sua introdução, podendo chegar mesmo a danificar o arranjo de eletrodos. Desta forma, aplicações interessantes para 0 sistema proposto podem ser 0 estudo de salinização de áreas agrícolas, onde normalmente se tem solo arado e fofo, ou estudo de contaminações rasas em litologias predominantemente arenosas.

Uma evolução do sistema que pode ser interessante é a instalação de vários segmentos com o arranjo Wenner, colocados em distâncias fixas em uma mesma sonda, e utilizá-la como sistema de monitoramento multinível, para isso simplesmente chaveando as alimentações (eletrodos AB) e os canais de leitura (eletrodos MN).

\section{AGRADECIMENTOS}

Os autores expressam seus agradecimentos à Fundação de Amparo à Pesquisa do Estado de São Paulo - FAPESP (Processo 0308714-0) e ao Conselho Nacional de Desenvolvimento Científico e Tecnológico - CNPq pela bolsa de Iniciação Científica do quinto autor, e ao Centro de Pesquisas em Águas Subterrâneas - CEPASUSP pela cessão da infra-estrutura laboratorial. 


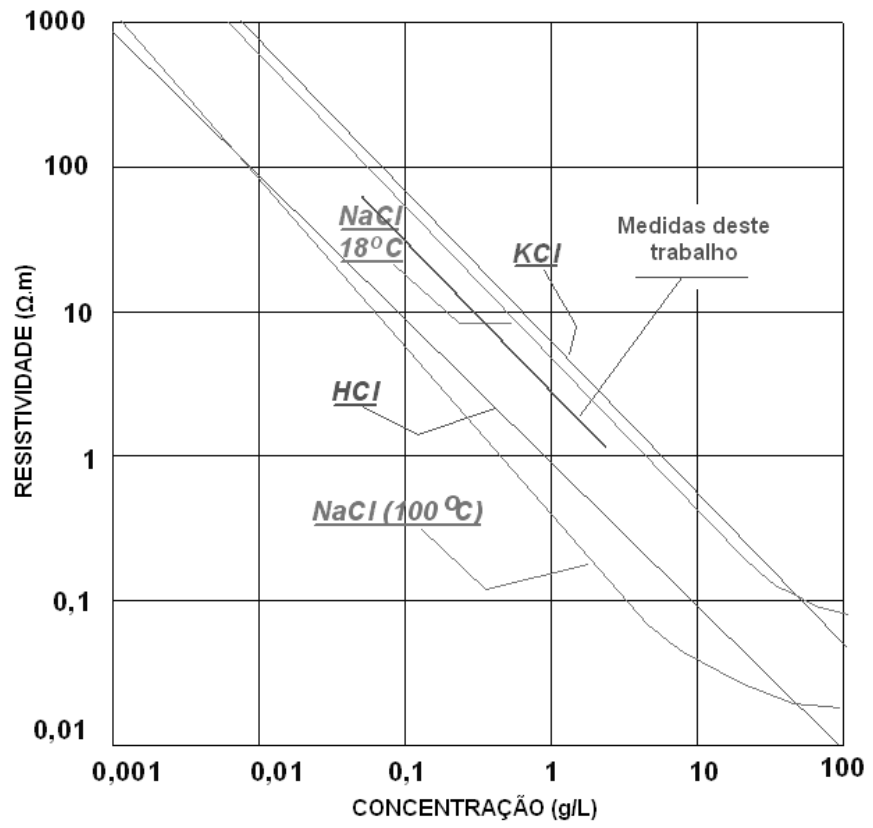

Figura 10 - Relação entre resistividade e concentração de sais em solução à temperatura de $18^{\circ} \mathrm{C} \mathrm{e} \mathrm{NaCl}$ também a $100^{\circ} \mathrm{C}$ (adaptado de Keller \& Frischknecht, 1977), incluindo os dados obtidos neste trabalho.

\section{REFERÊNCIAS}

GALLAS JDF. 2000. Principais Métodos Geoelétricos e suas Aplicações em Prospecção Mineral, Hidrogeologia, Geologia de Engenharia e Geologia Ambiental. Rio Claro. Tese (Doutorado em Geociências e Meio Ambiente) - Instituto de Geociências e Ciências Exatas, Universidade Estadual Paulista. $174 \mathrm{p}$.

KELLER GV \& FRISCHKNECHT FC. 1977. Electrical methods in geophysical prospecting. Oxford: Pergamon Press, $517 \mathrm{p}$.

ORELLANA E. 1972. Prospección geoeléctrica en corriente continua.
Madrid: Paraninfo, 523 p.

STIEGLITZ T. 2003. Measurements of Bulk Ground Conductivity at Sites of Submarine Groundwater Discharge, and Comparison with Other Methods. Preliminary Report to the Intergovernmental Oceanographic Commission and the International Hydrological Program on the IOC/IHP/IAEA Submarine Groundwater Discharge Intercomparison Experiment, Ubatuba, Brazil, 17 to 21 November 2003. pp. 17-26.

TELFORD WM, GELDART LP \& SHERIFF RE. 1990. Applied geophysics. Second Edition. Cambridge: Cambridge University Press, 770 p.

\section{NOTAS SOBRE OS AUTORES}

Fabio Taioli. Formou-se em Geologia pela USP em 1973. Trabalhou como geofísico da Petrobras até 1975 quando se transferiu para 0 Instituto de Pesquisas Tecnológicas do Estado de São Paulo - IPT. MSc em Mining Engineering pela The Pennsylvania State University na área de Geomecânica em 1987. Doutor pelo Instituto de Geociências da USP em 1992 na área de Geofísica Aplicada. Ingressou no IG-USP em 1995, tornando-se Livre-Docente (Associado) em 1999. Sua área de interesse é a aplicação de métodos geofísicos a problemas de engenharia civil e de minas e meio ambiente. É membro da SBGf, SEG, EEGS, ISRM, ABGE, IAEG, ABMS e CBMR.

José Domingos Faraco Gallas. Bacharelou-se em Geologia pela Universidade Federal do Rio Grande do Sul em 1978. Concluiu mestrado em Geofísica em 1990 pela Universidade Federal do Pará. Doutorou-se em Geociências e Meio Ambiente, ênfase em Geofísica Aplicada pela UNESP - Universidade Estadual Paulista em 2000. Foi Pesquisador do Instituto de Pesquisas Tecnológicas do Estado de São Paulo - IPT de 1979 até início de 2002. Desde fevereiro de 2002 é Prof. Doutor do Instituto de Geociências da Universidade de São Paulo - USP. Áreas de interesse: geofísica aplicada à prospecção mineral, hidrogeologia, geologia de engenharia e geologia ambiental.

Vilmondes Ribeiro. Formou-se em Engenharia Eletrônica, pela Universidade Mackenzie, em 1981. Ingressou no Instituto de Pesquisas Tecnológicas do Estado de São Paulo - IPT em 1982, onde se encontra até o presente. Inicialmente, como engenheiro de campo, atuou em Controle de Qualidade de sismógrafos aplicado à engenharia. Depois, atuou no desenvolvimento de diversos instrumentos e de metodologias, principalmente de acelerômetros, sismógrafos, e sistemas de aquisição de dados em campo. Áreas de interesse: ensaios e instrumentação aplicado às áreas de mecânica e hidráulica de rochas, geofísica e geologia de engenharia. 
Patrícia Braga Toledo lezzi. Formou-se em Geologia pela Universidade de São Paulo em 1999 e atua, desde 2000, na área de hidrogeologia e avaliação, diagnóstico, análise de risco e remediação de áreas contaminadas. Atualmente trabalha na empresa ENSR. É Mestranda do Programa de Pós-Graduação em Recursos Minerais e Hidrogeologia do Instituto de Geociências da Universidade de São Paulo.

Diego Potomati Vaccaro Nascimento. Estudante de Geologia da Universidade de São Paulo desde 2004. Desenvolveu em seu projeto de Iniciação Científica (CNPq-PIBIC 2005/2006) parte do projeto em questão. Áreas de interesse: geologia de engenharia, geologia ambiental e geofísica aplicada. 